\title{
Subgrid parameterisation of the eddy-meanfield interactions in a baroclinic quasi-geostrophic ocean
}

\author{
V. Kitsios ${ }^{1} \quad$ J. S. Frederiksen ${ }^{2} \quad$ M. J. Zidikheri ${ }^{3}$
}

(Received 31 October 2012; revised 31 May 2013)

\begin{abstract}
We present parameterisations of the subgrid eddy-eddy and eddymeanfield interactions in a baroclinic ocean representative of the Antarctic Circumpolar Current. Benchmark direct numerical simulations were undertaken using a quasi-geostrophic spectral spherical harmonic code of maximum zonal and total truncation wavenumber of $\mathrm{T}=252$. The eddy-eddy interactions are represented by both stochastic and deterministic parameterisations, with model coefficients determined from the direct numerical simulations truncated back to the large eddy simulation truncation wavenumber $T_{R}$ less than $T$. Coefficients of the deterministic eddy-meanfield model are determined by a new least squares regression method. Truncations were repeated for various $T_{R}$,
\end{abstract}

http://journal.austms.org.au/ojs/index.php/ANZIAMJ/article/view/6309 gives this article, (c) Austral. Mathematical Soc. 2013. Published August 16, 2013, as part of the Proceedings of the 16th Biennial Computational Techniques and Applications Conference. ISSN 1446-8735. (Print two pages per sheet of paper.) Copies of this article must not be made otherwise available on the internet; instead link directly to this URL for this article. 
with the dependence of the coefficients on $T_{R}$ identified. Kinetic energy spectra from the large eddy simulations using these coefficients agree with the direct numerical simulations.

Subject class: 76F65, 76F30

Keywords: large eddy simulation, subgrid parameterisation, turbulence, ocean

\section{Contents}

1 Introduction

2 Direct numerical simulation

3 Subgrid parameterisations

C465

3.1 Subgrid eddy-eddy parameterisation . . . . . . . . . . C466

3.2 Subgrid eddy-meanfield parameterisation . . . . . . . . C468

4 Large eddy simulation

$\mathrm{C} 472$

5 Concluding remarks

C472

References

$\mathrm{C} 473$

\section{Introduction}

As it is not possible to explicitly resolve all of the scales of motion in the ocean, one resorts to large eddy simulations (LES), where the large eddies are resolved by a computational grid and the unresolved subgrid interactions are parameterised. There are four main types of subgrid interactions: eddyeddy; eddy-meanfield; eddy-topographic; and meanfield-meanfield [3]. If these interactions are not self-consistently parameterised, then an increase in 
resolution will not necessarily increase the accuracy of the explicitly resolved scales. This dependence of the resolved scales on resolution has been an issue in general circulation models (GCMs) since the earliest simulations of weather and climate, and persists today in even the most sophisticated GCMs [8].

The subgrid eddy-eddy interactions represent the interactions between the transient resolved and subgrid eddies. These interactions are typically modelled by a dissipation operator acting on the resolved field. There is at present no fundamental theory governing the properties of the dissipation, with simulations generally tuned to yield desirable properties and to achieve numerical stability [6]. In early oceanic simulations these empirical dissipation operators were applied in horizontal and vertical directions [1]. Redi proposed [9] that the dissipation operator should be diagonal when oriented in isopycnal (constant density) directions, effectively setting all diapycnal subgrid interactions to zero. Whatever the coordinate system, the dissipation operator is typically isotropic and has a form which is either of Laplacian or bi-harmonic order [6]. However, recently Kitsios et al. [7] developed a set of scaling laws governing how the dissipation changes with resolution. The dissipation strength and order are functions of resolution, and at typical resolutions the dissipation is of higher order than bi-harmonic.

The eddy-meanfield interactions represent the interactions between the subgrid eddies and the resolved meanfield. The eddy-meanfield parameterisations of Gent and McWilliams [5] is one of the most commonly adopted in simulations of the ocean. This parameterisation prescribes the structure of the skew symmetric elements of the dissipation operator in isopycnal coordinates acting on the temperature in the energy equation. The addition of the skew symmetric terms produces simulations with smaller, more realistic diapycnal fluctuations [6]. There is currently no analogous approach accounting for the eddy-meanfield interactions in the momentum equations.

The parameterisation of the quasi-geostrophic eddy-meanfield interactions is the main focus of the current study. Specifically, we develop parameterisations for a flow representative of the Antarctic Circumpolar Current (ACC), gener- 
ated using a quasi-geostrophic spectral spherical harmonic code of maximum zonal and total truncation wavenumber of $T=252$. The eddy viscosity coefficients are calculated from the statistics of high resolution reference direct numerical simulations (DNSs) truncated back to the LES truncation wavenumber $T_{R}<T$. The coefficients representing the eddy-eddy interactions are determined using the method of Frederiksen and Kepert [4]. This approach was previously successfully applied to quasi-geostrophic (QG) simulations of the ocean, comprising of sheared currents, Rossby waves, and baroclinic instability $[7,10,11]$. The eddy-meanfield interactions are determined from the DNS statistics via a new approach published here for the first time.

Section 2 summarises the QG potential vorticity equation (QGPVE), and presents the resulting DNS flow fields and spectra. DNS in this content refers to a high resolution reference simulation, and not one that resolves down to the Kolmogorov scale. Section 3 shows how the subgrid eddy-eddy and eddy-meanfield coefficients are determined from the DNS, along with the subgrid coefficients themselves. Section 4 outlines the LES version of the QGPVE, and the spectra from the LES are compared to that of the reference DNS. Concluding remarks are presented in Section 5.

\section{Direct numerical simulation}

We employ the two level QG model of Frederiksen [2], which captures the essential dynamics of baroclinic and barotropic instabilities. The vorticity is represented at two vertical levels, with $j=1$ representing a depth of approximately $200 \mathrm{~m}$, and $j=2$ a depth of $600 \mathrm{~m}$. The system is nondimensionalised by using the radius of the Earth $(a=6371 \mathrm{~km})$ as a length scale, and the inverse of the Earth's angular velocity $\left(\Omega=7.292 \times 10^{-5} \mathrm{~s}^{-1}\right)$ as a time scale. By default all variables are assumed to be nondimensional unless units are specified.

The QGPVE is spectrally discretised by expanding the field variables in 
spherical harmonics with the zonal (longitudinal) wavenumber $\mathfrak{m}$ and the total wavenumber $n$. This results in the prognostic equations for the spectral coefficients of the potential vorticity, $q_{m n}^{j}=\zeta_{m n}^{j}+(-1)^{j} F_{L}\left[\psi_{m n}^{1}-\psi_{m n}^{2}\right]$, where $\zeta_{m n}^{j}=-n(n+1) \psi_{m n}^{j}$ are the spectral coefficients of the vorticity, $\psi_{m n}^{j}$ are the streamfunction coefficients, $n(n+1)$ is the discrete form of the Laplacian, and the superscript $j$ on the flow variables denotes the level. Here $F_{L}$ is a layer coupling parameter, related to the Rossby radius of deformation by $r_{R}=1 / \sqrt{2 F_{L}}$. The evolution equation for $q_{m n}^{j}$ is

$$
\begin{aligned}
\frac{\partial q_{m n}^{j}}{\partial t}= & i \sum_{p, q} \sum_{r, s} \kappa_{n q s}^{m p r} \psi_{-p q}^{j} q_{-r s}^{j}-i \omega_{m n} \zeta_{m n}^{j}-\alpha^{j}(n) \zeta_{m n}^{j} \\
& +\kappa_{n}\left(\tilde{q}_{m n}^{j}-q_{m n}^{j}\right)-n(n+1) \sum_{l=1}^{2} v_{0}^{j l}(m, n) q_{m n}^{l} .
\end{aligned}
$$

The summations in the first term are over the triangular wavenumber set $\mathbf{T}=\mathbf{C}(\mathbf{T})$, with $\mathbf{T}$ the DNS truncation wavenumber and

$$
\mathbf{C}(\mathrm{T})=[\mathrm{p}, \mathrm{q}, \mathrm{r}, \mathrm{s}] \quad \text { for }-\mathrm{T} \leqslant \mathrm{p}, \mathrm{r} \leqslant \mathrm{T}, \quad|\mathrm{p}| \leqslant \mathrm{q} \leqslant \mathrm{T}, \quad|\mathrm{r}| \leqslant s \leqslant \mathrm{~T} .
$$

The Rossby wave frequency is $\omega_{m n}=-B m /[n(n+1)]$, where $B=2$ with the chosen nondimensionalisation. The drag at each level is the function $\alpha^{j}(n)=\alpha_{\max }^{j}\{1-\operatorname{erf}[0.1(n-50)]\} / 2$, where erf is the error function and the damping times $1 / \alpha_{\max }^{j}$ are 40 days for level one $(j=1)$ and 10 days for level two $(j=2)$. The interaction coefficients $K_{\text {nqs }}^{m p r}$ were discussed in detail by Frederiksen and Kepert [4]. All simulations are driven toward a mean state $\tilde{\mathbf{q}}_{m \mathfrak{n}}^{j}$ that is purely zonal $\left(\tilde{\mathbf{q}}_{\mathfrak{m} n}^{j}\right.$ are zero unless $\mathrm{m}=0$ ) and corresponds to a large scale easterly current in the mid-latitudes of the southern hemisphere, broadly representative of the ACC. The simulations are driven toward this state by a relaxation parameter $\kappa_{n}$, which for $m=0$ and $n \leqslant 15$ is $\kappa_{n}=10^{-6} s^{-1}$, and $\kappa_{n}=0$ for all remaining wavenumber pairs. The bare eddy viscosity $v_{0}^{j l}(m, n)$ is necessary as the DNS does not resolve all of the scales of motion. We represent $v_{0}^{j l}(m, n)$ in its general anisotropic matrix form (dependent on $m$ and $\mathfrak{n}$ ) but in our simulations it is isotropic 


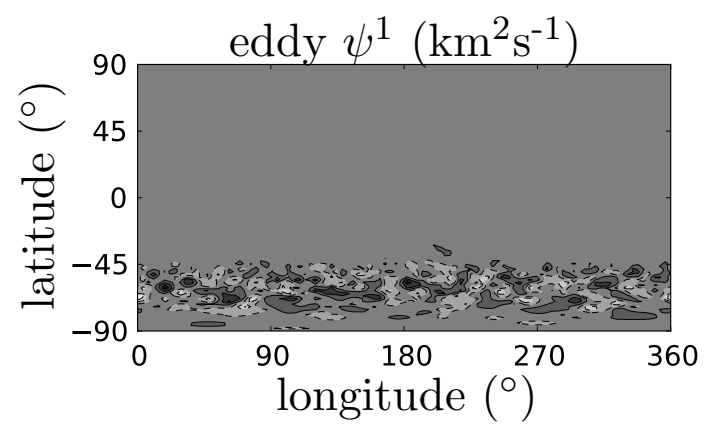

(a)

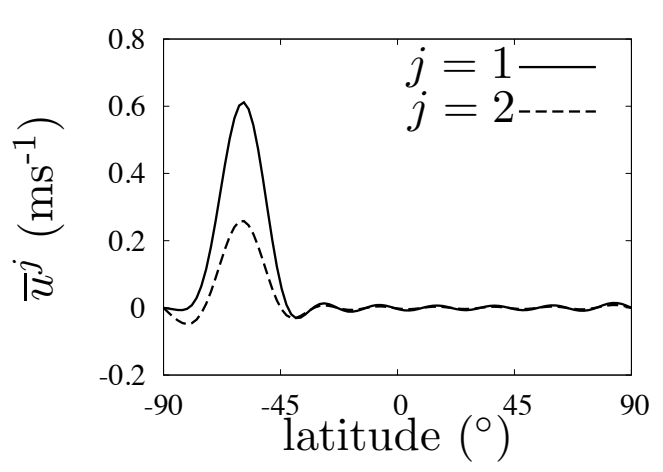

(b)

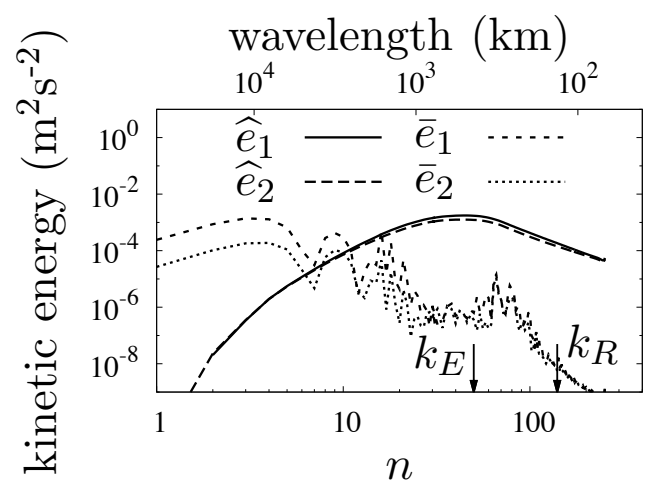

(c)

Figure 1: DNS flow field: (a) level one instantaneous eddy (nonzonal) streamfunction field $\left(\psi^{1}\right)$ from contours level $-1.4 \mathrm{~km}^{2} \mathrm{~s}^{-1}$ (black) to $1.4 \mathrm{~km}^{2} \mathrm{~s}^{-1}$ (white); (b) time averaged zonal current $\left(\overline{\mathfrak{u}}^{j}\right)$ at level $\mathfrak{j}$; and (c) mean $\left(\bar{e}_{j}\right)$ and fluctuating $\left(\widehat{e}_{j}\right)$ kinetic energy spectra at level $j$.

(dependent only on $n$ ) where $v_{0}^{j l}(m, n)=v_{0}^{j l}(n)=\delta_{l j} v_{0}^{j j}(T)[n / T]_{0}^{\rho_{0}^{j}-2}$, and $\delta_{l j}$ is the Kronecker delta function, ensuring the off diagonal elements are zero. Here $\gamma_{0}^{\mathrm{jj}}(\mathrm{T})$ is the value of the diagonal elements at truncation and the exponent $\rho_{0}^{j}$ determines the steepness of $\nu_{0}^{\mathrm{jj}}(\mathrm{n})$. There is no topography or orography in the present simulations. 
A DNS of a QG ocean is undertaken with $\mathrm{F}_{\mathrm{L}}=2.5 \times 10^{-10} \mathrm{~m}^{-2}$, corresponding to a Rossby radius of $r_{R}=1 / \sqrt{2 F_{L}}=45 \mathrm{~km}$. The nondimensional Rossby wavenumber is $k_{R}=a / r_{R}=142$, which is consistent with the simulations of Zidikheri and Frederiksen [10]. Here we use a DNS truncation wavenumber of $\mathrm{T}=252$, which is equivalent to 768 longitudinal and 384 latitudinal grid points, or a grid point approximately every 0.5 degrees. The time step size used is $\Delta t=1200 \mathrm{~s}$, and the statistics are accumulated over a period of 100 years. A snapshot of the level one instantaneous streamfunction field $\psi^{1}$ minus the zonal component is shown in Figure 1(a), which illustrates that the dominant structures are located in the mid to high latitudes of the southern hemisphere, consistent with the ACC. The corresponding time averaged zonal current $\bar{u}^{j}$ is shown as function of latitude in Figure 1(b). The maximum velocities of the time averaged current at depths of $200 \mathrm{~m}$ and $600 \mathrm{~m}$ are approximately $0.6 \mathrm{~ms}^{-1}$ and $0.3 \mathrm{~ms}^{-1}$, respectively. Further details on the basic state were presented by Kitsios et al. [7]. The kinetic energy spectrum $e_{j}$ is decomposed into mean $\left(\bar{e}_{j}\right)$ and transient $\left(\widehat{e}_{j}\right)$ energies. Figure 1(c) illustrates that the level one energy is greater than the level two energy for all $n$. The scale wavenumber $\mathrm{k}_{\mathrm{E}} \approx 70$ is defined as the wavenumber at which the self similar inertial range begins. It is labelled on the $\boldsymbol{n}$ axis of Figure 1(c) along with the Rossby wavenumber $k_{R}$.

\section{Subgrid parameterisations}

The resolution of a LES is lower than the associated DNS and confined to the resolved scale wavenumber set $\mathbf{R}=\mathbf{C}\left(T_{R}\right)$, where $T_{R}$ is the LES truncation wavenumber such that $T_{R}<T$. The subgrid wavenumber set is defined as $\mathbf{S}=\mathbf{T}-\mathbf{R}$. To facilitate a discussion on the flow decomposition we let $\mathbf{q}=\left(\mathbf{q}_{\mathrm{mn}}^{1}, \mathbf{q}_{\mathrm{mn}}^{2}\right)^{\top}$ for a given wavenumber pair. In this vector notation $\mathbf{q}_{t}(t)=\mathbf{q}_{t}^{\mathbf{R}}(\mathbf{t})+\mathbf{q}_{t}^{\mathbf{S}}(\mathbf{t})$, where $\mathbf{q}_{\mathrm{t}}$ is the tendency (time derivative) of $\mathbf{q}$. The tendency of the resolved scales is $\mathbf{q}_{t}^{\mathbf{R}}$, where all triadic interactions involve wavenumbers less than $T_{R}$. The remaining subgrid tendency $\mathbf{q}_{\mathbf{t}}^{\mathbf{S}}$ has at least 
one wavenumber greater than $T_{R}$ which is involved in the triadic interactions. On decomposing $\mathbf{q}_{\mathrm{t}}^{\mathbf{S}}$,

$$
\mathbf{q}_{t}^{\mathbf{S}}(\mathbf{t})=\widehat{\mathbf{q}}_{\mathbf{t}}^{\mathbf{S}}(\mathrm{t})+\overline{\mathbf{f}}
$$

where $\widehat{\mathbf{q}}_{\mathbf{t}}^{\mathbf{S}}$ is the fluctuating component representing the eddy-eddy interactions and $\overline{\mathbf{f}} \equiv\left\langle\mathbf{q}_{\mathbf{t}}^{\mathbf{S}}\right\rangle$ is the ensemble averaged subgrid tendency representing the eddy-meanfield interactions. The parameterisation of both of these interaction types are determined from the statistics of the reference DNS.

\subsection{Subgrid eddy-eddy parameterisation}

The $\widehat{\mathbf{q}}_{\mathbf{t}}^{\mathbf{S}}$ is represented by the stochastic equation

$$
\widehat{\mathbf{q}}_{\mathrm{t}}^{\mathbf{S}}(\mathrm{t})=-\mathrm{D}_{\mathrm{d}} \widehat{\mathbf{q}}(\mathrm{t})+\widehat{\mathbf{f}}(\mathrm{t})
$$

where $D_{d}$ is the subgrid drain dissipation matrix, $\widehat{\mathbf{q}}$ is the fluctuating component of $\mathbf{q}$, and $\widehat{\mathbf{f}}$ is a random forcing vector. As our simulations have two vertical levels, $D_{d}$ is a time independent $2 \times 2$ matrix, and $\widehat{\mathbf{f}}$ is a time dependent two element column vector. The $D_{d}$ matrix is determined by post-multiplying both sides of (4) by $\widehat{\mathbf{q}}^{\dagger}\left(\mathbf{t}_{0}\right)$, integrating over the turbulent decorrelation period $\tau$, ensemble averaging to remove the contribution of $\widehat{\mathbf{f}}$, and rearranging to produce

$$
D_{d}=-\left\langle\int_{t_{0}}^{t_{0}+\tau} \widehat{\mathbf{q}}_{t}^{S}(\sigma) \widehat{\mathbf{q}}^{\dagger}\left(t_{0}\right) d \sigma\right\rangle\left\langle\int_{t_{0}}^{t_{0}+\tau} \widehat{\mathbf{q}}(\sigma) \widehat{\mathbf{q}}^{\dagger}\left(t_{0}\right) d \sigma\right\rangle^{-1},
$$

where $\dagger$ denotes the Hermitian conjugate of vectors and matrices. The angled brackets denote ensemble averaging, with each ensemble member determined by shifting $t_{0}$ forward by one time step. The turbulence decorrelation time $\tau$ is chosen to be sufficiently large to capture the memory effects.

The model for $\widehat{\mathbf{f}}$ is determined by calculating the Hermitian matrix $\mathcal{F}_{\mathrm{b}}=$ $F_{b}+F_{b}^{\dagger}$, where $F_{b}=\left\langle\widehat{\mathbf{f}}(t) \widehat{\mathbf{q}}^{\dagger}(t)\right\rangle$. Post-multiplying both sides of (4) by $\widehat{\mathbf{q}}^{\dagger}\left(t_{0}\right)$ 
and adding the conjugate transpose of (4) pre-multiplied by $\widehat{\mathbf{q}}\left(\mathbf{t}_{0}\right)$ yields the Lyapunov equation

$$
\left\langle\widehat{\mathbf{q}}_{\mathrm{t}}^{\mathbf{S}}(\mathrm{t}) \widehat{\mathbf{q}}^{\dagger}(\mathrm{t})\right\rangle+\left\langle\widehat{\mathbf{q}}(\mathrm{t}) \widehat{\mathbf{q}}_{\mathrm{t}}^{\mathbf{S}^{\dagger}}(\mathrm{t})\right\rangle=-\mathrm{D}_{\mathrm{d}}\left\langle\widehat{\mathbf{q}}(\mathrm{t}) \widehat{\mathbf{q}}^{\dagger}(\mathrm{t})\right\rangle-\left\langle\widehat{\mathbf{q}}(\mathrm{t}) \widehat{\mathbf{q}}^{\dagger}(\mathrm{t})\right\rangle \mathrm{D}_{\mathrm{d}}^{\dagger}+\mathcal{F}_{\mathrm{b}} .
$$

Given that $D_{d}$ is known, $\mathcal{F}_{b}$ can now be calculated. At this point the formulation is general, and $\widehat{\mathbf{f}}$ is coloured noise. However, for the implementation of the stochastic subgrid parameterisation it is sufficient to assume that $\widehat{\mathbf{f}}$ is the white noise process for which $\left\langle\widehat{\mathbf{f}}(\mathrm{t}) \widehat{\mathbf{f}}^{\dagger}\left(\mathrm{t}^{\prime}\right)\right\rangle=\mathcal{F}_{\mathrm{b}} \delta\left(\mathrm{t}-\mathrm{t}^{\prime}\right)$.

The subgrid model in (4) represents the subgrid scales in a stochastic manner. One can also do so deterministically, where the subgrid tendency is modelled according to $\widehat{\mathbf{q}}_{\mathrm{t}}^{\mathbf{S}}(\mathrm{t})=-\mathrm{D}_{\text {net }} \widehat{\mathbf{q}}(\mathrm{t})$ with the net dissipation $\mathrm{D}_{\text {net }}=\mathrm{D}_{\mathrm{d}}+\mathrm{D}_{\mathrm{b}}$ and the backscatter dissipation $D_{b}=-F_{b}\left\langle\widehat{\mathbf{q}}(t) \widehat{\mathbf{q}}^{\dagger}(t)\right\rangle^{-1}$. Here the subgrid coefficients are presented in eddy viscosity form, where the drain, backscatter and net eddy viscosities are related to their respective dissipations by $v_{\mathrm{d}} \equiv$ $D_{d} /[n(n+1)], v_{b} \equiv D_{b} /[n(n+1)]$ and $v_{n e t} \equiv D_{\text {net }} /[n(n+1)]$.

We now present the subgrid model coefficients from the DNS statistics presented in Section 2. The DNS is truncated to various values of $T_{R}$ to determine how the eddy viscosities change with resolution. Firstly we present the anisotropic drain eddy viscosity $v_{d}$ truncated to $T_{R}=126$, with $\tau=288 \Delta t=$ 4 days. The real component of the upper diagonal $v_{d}^{11}(m, n)$ is illustrated in Figure 2(a). At this resolution $v_{d}^{11}(m, n)$ increases with $n$, and for $m<60$ has only a weak dependence on $m$ and hence is approximately isotropic in this region. The lower diagonal element $v_{d}^{22}(m, n)$ has a similar form, and the off diagonal elements are small in comparison. These observations are also true for the backscatter and net eddy viscosities. We also find that $v_{\mathrm{d}} \approx 2 v_{\text {net }} \approx-2 v_{\mathrm{b}}$. However, at lower truncation levels the coefficients become more anisotropic and the off diagonal elements become proportionally more important. The self similarity of the eddy viscosities is most clearly illustrated by the isotropised (averaged over $\mathrm{m}$ ) profiles. The real component of the upper diagonal isotropised drain eddy viscosity $v_{d}^{11}(n)$ is illustrated in Figure 2(b) for various truncation levels. As the resolution increases 


$$
R e\left[\nu_{d}^{11}(m, n)\right] \times 10^{6}
$$

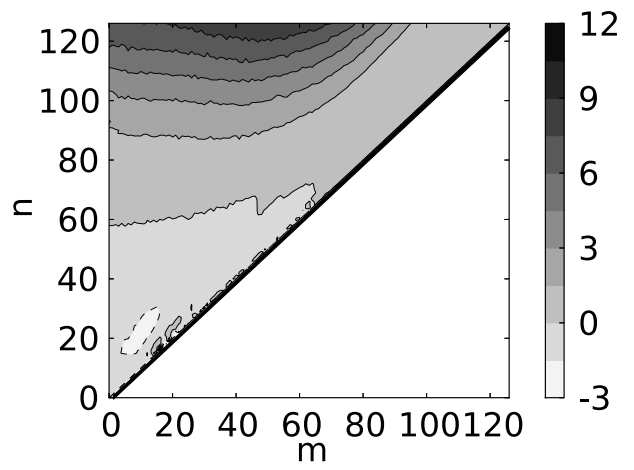

(a)

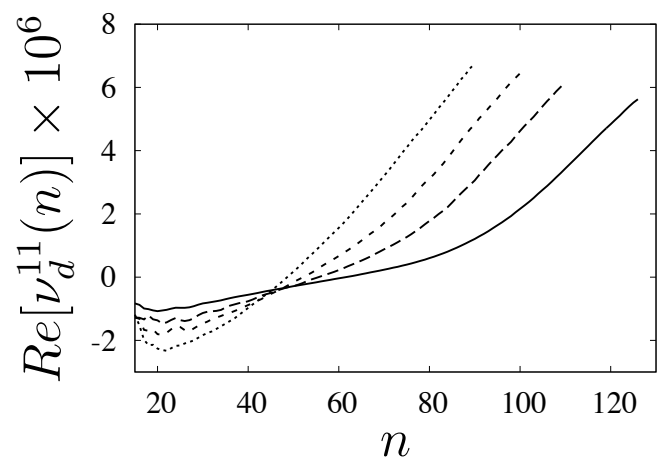

(b)

Figure 2: Subgrid eddy-eddy parameterisation coefficients for truncations of a $T=252$ DNS. (a) Anisotropic coefficients for an LES with $T_{R}=126$; and (b) isotropic coefficients for $T_{R}=90$ (dot),100 (small dash), 110 (large dash) and 126 (solid).

the maximum value of $\operatorname{Re}\left[v_{d}^{11}(n)\right]$ decreases, and the most negative value approaches zero. For truncations with $T_{R}>k_{E}$ the positive values of the eddy viscosities are concentrated in the last $k_{E}$ wavenumbers before truncation. This means that when plotted against $n / T_{R}$ the profiles become steeper as the resolution increases. Scaling laws governing how $v_{d}, v_{b}$ and $v_{\text {net }}$ change with resolution, and the off diagonal elements were presented in more detail by Kitsios et al. [7].

\subsection{Subgrid eddy-meanfield parameterisation}

The parameterisation of the eddy-meanfield term represents the relationship between the ensemble averaged subgrid tendency $\overline{\mathbf{f}}$ and the ensemble averaged 
field $\langle\mathbf{q}\rangle$. For each wavenumber pair we assume the functional form

$$
\overline{\mathbf{f}}=-\overline{\mathrm{D}}\langle\mathbf{q}\rangle+\mathbf{b},
$$

where $\overline{\mathrm{D}}$ is a $2 \times 2$ dissipation operator and offset $\mathbf{b}$ is a constant two element vector. We assume that (7) also holds for small perturbations of the climate centred about the ensemble averaged climate, such that

$$
\overline{\mathbf{f}}_{\mathrm{i}}=-\overline{\mathrm{D}} \overline{\mathbf{q}}_{\mathrm{i}}+\mathbf{b}+\boldsymbol{\epsilon}_{\mathrm{i}}
$$

where $\overline{\mathbf{q}}_{i}$ and $\overline{\mathbf{f}}_{i}$ are the time averaged meanfield and subgrid tendency calculated over the ith non-overlapping time window of length $\tau_{M}$, and $\boldsymbol{\epsilon}_{\mathfrak{i}}$ is the associated two element error vector. The ensemble averages of each of the terms are $\left\langle\overline{\mathbf{q}}_{\boldsymbol{i}}\right\rangle \equiv\langle\mathbf{q}\rangle,\left\langle\overline{\mathbf{f}}_{\mathfrak{i}}\right\rangle \equiv \overline{\mathbf{f}}$ and $\left\langle\boldsymbol{\epsilon}_{\mathfrak{i}}\right\rangle=0$. The dissipation is solved in a least squares sense by subtracting (7) from (8), post-multiplying by $\left(\overline{\mathbf{q}}_{i}-\langle\mathbf{q}\rangle\right)^{\dagger}$, ensemble averaging both sides, and rearranging for $\overline{\mathrm{D}}$ to produce

$$
\overline{\mathrm{D}}=-\left(\left\langle\overline{\mathbf{f}}_{i} \overline{\mathbf{q}}_{i}^{\dagger}\right\rangle-\overline{\mathbf{f}}\langle\mathbf{q}\rangle^{\dagger}\right)\left(\left\langle\overline{\mathbf{q}}_{i} \overline{\mathbf{q}}_{i}^{\dagger}\right\rangle-\langle\mathbf{q}\rangle\langle\mathbf{q}\rangle^{\dagger}\right)^{-1}
$$

where we assume that the error term $\boldsymbol{\epsilon}_{i}$ is uncorrelated with $\left(\overline{\mathbf{q}}_{i}-\langle\mathbf{q}\rangle\right)^{\dagger}$. Once $\bar{D}$ is known we can determine the offset by rearranging (7) for $\mathbf{b}$.

The above process is applied to the DNS with $T=252$. The only mechanism for symmetry breaking in (1) is the forcing term $\kappa_{n}\left(\tilde{q}_{m n}-q_{m n}\right)$. As $\kappa_{n}$ is only nonzero for $m=0$ and $n \leqslant 15$, it is only the $\tilde{f}_{m n}^{j}$ and $\bar{q}_{m n}^{j}$ at these wavenumber components that are nonzero under sufficient sampling. The average subgrid tendency $\overline{\mathbf{f}}$ is plotted in Figure 3(a) for a truncation level $T_{R}=126$. For all truncation levels $\bar{f}_{0 n}^{1}$ is approximately a mirror image of $\bar{f}_{0 n}^{2}$, which modifies the mean shear in the flow. It therefore makes more sense to present $\overline{\mathbf{f}}$ in baroclinic space. We define $\overline{\mathbf{f}}^{\mathrm{B}} \equiv\left(\overline{\mathbf{f}}_{\mathfrak{m} \mathfrak{n}}^{\mathrm{BT}}, \overline{\mathrm{f}}_{\mathfrak{m} \mathfrak{n}}^{\mathrm{BC}}\right)^{\mathrm{T}}$, where $\overline{\mathbf{f}}_{\mathfrak{m} \mathfrak{n}}^{\mathrm{BT}}$ is the barotropic component and $\bar{f}_{m \mathfrak{n}} \mathrm{BC}$ is the baroclinic component, which we find to be dominant for the present data. The conversion between the coordinate systems is $\overline{\mathbf{f}}_{\mathrm{B}}=\mathrm{B} \overline{\mathbf{f}}$, where

$$
\mathrm{B}=\frac{1}{2}\left[\begin{array}{cc}
1 & 1 \\
\frac{1}{c_{n}} & -\frac{1}{c_{n}}
\end{array}\right]
$$




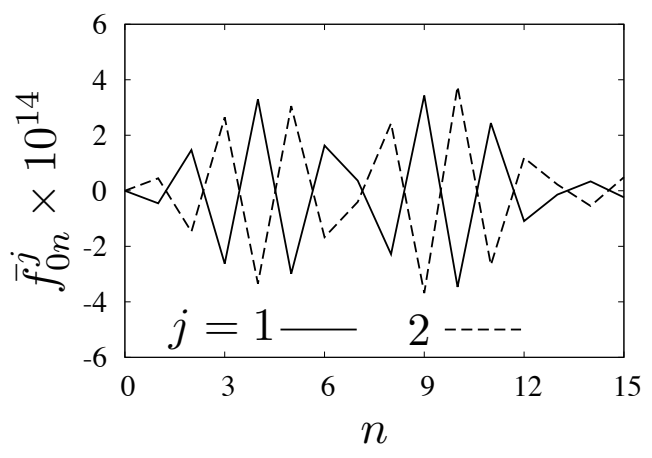

(a)

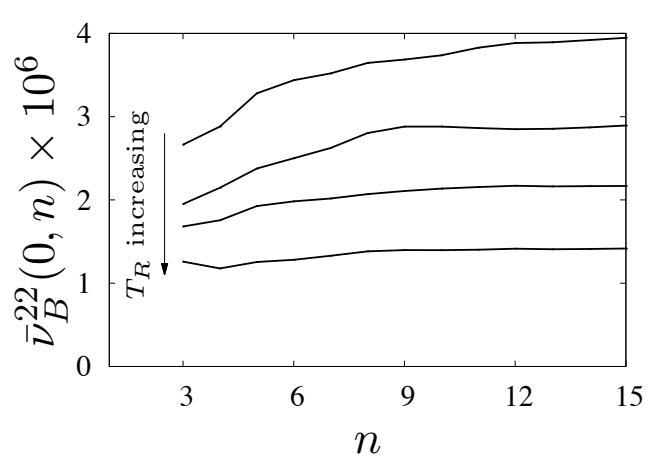

(c)

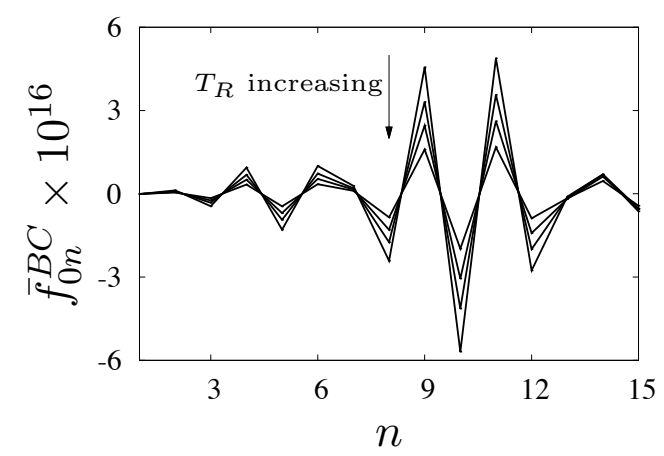

(b)

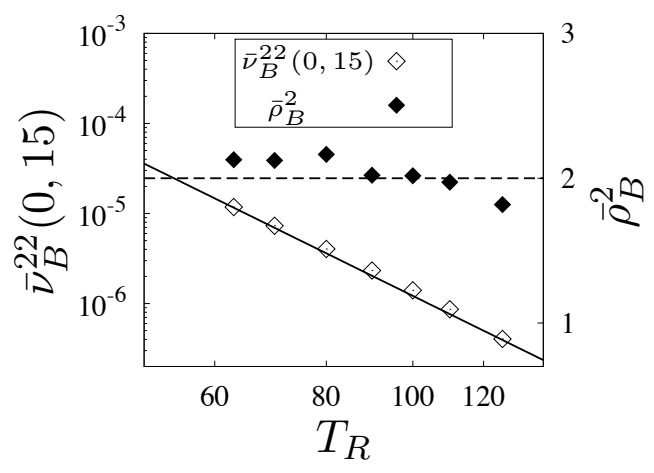

(d)

Figure 3: Subgrid eddy-meanfield parameterisation coefficients for truncations of a $T=252$ DNs. (a) Mean potential vorticity tendency $\vec{f}_{\text {on }}^{j}$ for an LES with $T_{R}=126$. For $T_{R}=90,100,110,120$ : (b) mean baroclinic tendency $\bar{f}_{0 n}^{B C}$; and (c) eddy viscosity component $\bar{v}_{B}^{22}$. (d) For various $T_{R}$, the amplitude, $\bar{v}_{\mathrm{B}}^{22}(0,15)$, and slope, $\bar{\rho}_{\mathrm{B}}^{2}$, of $\bar{\nu}_{\mathrm{B}}^{22}$. 
and $c_{n}=1+2 F_{L} /[n(n+1)]$. Subgrid truncations are repeated for lower values of $T_{R}$, with the dominant $\bar{f}_{0 n}^{B C}$ component illustrated in Figure 3(b). As the system is truncated more heavily ( $T_{R}$ decreasing), there are more subgrid eddy-meanfield interactions and consequently $\bar{f}_{\mathrm{On}}^{\mathrm{BC}}$ increases. For various truncation levels we calculate $\overline{\mathrm{D}}$ using (9), and scale it into eddy viscosity units using $\bar{v}=\bar{D} /[n(n+1)]$. The eddy viscosity is then transformed into barotropic/baroclinic space via $\bar{v}_{\mathrm{B}}=\mathrm{B} \overline{\mathrm{v}} \mathrm{B}^{-1}$. We find the $\bar{v}_{\mathrm{B}}^{22}$ component to be dominant, which represents the mean baroclinic tendency as a function of the mean baroclinic field. This component is plotted for various resolutions in Figure 3(c), illustrating that as the truncation level decreases the required eddy viscosity increases, consistent with the observations of $\bar{f}_{0 n}^{B C}$.

The change in magnitude, $\bar{v}_{\mathrm{B}}^{22}(0,15)$, and slope, $\bar{\rho}_{\mathrm{B}}^{2}$, of the baroclinic eddy viscosity term is quantified by least squares fitting the $\bar{v}_{B}^{22}(0, n)$ profiles to

$$
\bar{v}_{B}^{22}(0, n)=\bar{v}_{B}^{22}(0,15)(n / 15)^{\bar{\rho}_{B}^{2}-2},
$$

for $\mathrm{n} \leqslant 15$. In Figure $3(\mathrm{~d}), \overline{\boldsymbol{v}}_{\mathrm{B}}^{22}(0,15)$ is plotted as hollow diamonds on the left vertical axis against $T_{R}$, and $\bar{\rho}_{\mathrm{B}}^{2}$ is plotted as filled diamonds on the right vertical axis. The steepness, $\bar{\rho}_{\mathrm{B}}^{2}$, is relatively constant, $\bar{\rho}_{\mathrm{B}}^{2} \approx 2$, for all $T_{R}$. The data also illustrates that as $T_{R}$ increases (more scales resolved), the strength $\bar{v}_{B}^{22}(0,15)$ decreases. The strength of the eddy-meanfield eddy viscosity also decreases with resolution and this decrease is faster than the eddy viscosity representing the eddy-eddy interactions. This means that as the resolution decreases, the eddy-meanfield interactions become proportionally more important. The eddy-meanfield eddy viscosities are also found to be insensitive to the choice of window period $\tau_{M}$, as long as $\tau_{M}$ is greater than one week. Future work will address the sensitivity of these results to the Rossby wavenumber $k_{R}$, the target climate state $\tilde{\mathbf{q}}_{\mathbf{m} n}$, and the relation rate $\boldsymbol{k}_{n}$. 


\section{Large eddy simulation}

The LES equations are the same as for the DNS in (1), with $\left(q_{t}^{S}\right)_{m n}^{j}$ added to the right hand side, and solved over the wavenumber set $\mathbf{R}$ instead of $\mathbf{T}$. The fundamental form of $\left(q_{t}^{S}\right)_{m n}^{j}$ is the stochastic anisotropic representation

$$
\left(q_{t}^{s}\right)_{m n}^{j}=-\sum_{l=1}^{2} D_{d}^{j l}(m, n) \widehat{q}_{m n}^{l}+\widehat{f}_{m n}^{j}+\bar{f}_{m n}^{j} .
$$

In the deterministic form, $D_{d}^{j l}(m, n)$ is replaced with $D_{\text {net }}^{j l}(m, n)$ and $\widehat{f}_{m n}^{j}$ is removed. The kinetic energy spectra of the DNS is compared to the spectra from the LES variants at $T_{R}=126$. The spectra at level one $\left(e_{1}\right)$ of both LES variants are compared to $e_{1}$ of the DNS in Figure 4(a), with each spectra offset for clarity. Whilst the stochastic approach is the fundamental form, we find that the deterministic LES performs equally well, with both variants achieving excellent agreement with the DNS. In Figure 4(b) the spectra at level two $\left(e_{2}\right)$ of the LES is compared to $e_{2}$ of the DNS, with both variants again achieving excellent agreement.

\section{Concluding remarks}

Subgrid parameterisations were developed for the eddy-eddy and eddymeanfield interactions for an oceanic flow representative of the ACC. Deterministic and stochastic subgrid parameterisations were presented for the eddy-eddy interactions using the approach of Frederiksen and Kepert [4]. The stochastic variant consists of a drain eddy viscosity and a backscatter noise term. The deterministic version is governed solely by the net eddy viscosity, which represents the net effect of the drain and backscatter. The eddy viscosity matrices required for the eddy-meanfield parameterisation were calculated using a new approach presented here for the first time. In both approaches the wavenumber dependent eddy viscosity matrices were derived 


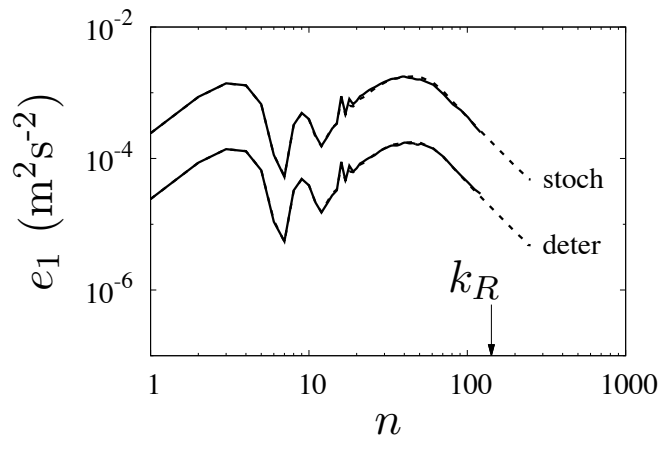

(a)

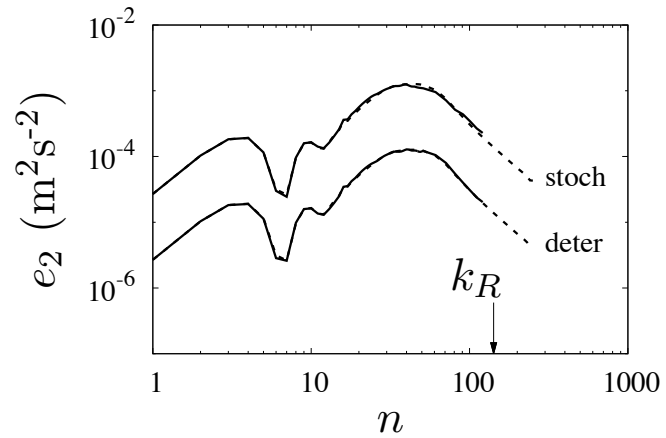

(b)

Figure 4: DNS kinetic energy spectra (dashed line) compared to LES (solid line) with anisotropic deterministic (deter) and stochastic (stoch) parameterisations at level: (a) one; and (b) two.

self-consistently from the statistics of higher resolution DNS. The kinetic energy spectra resulting from both LES variants agree with the spectra from the reference DNS, and was achieved without parameters or a tuning procedure. We hope to apply this subgrid modelling approach to more complex mutli-level primitive equation simulation codes incorporating more complete dynamics and physics.

Acknowledgements V. Kitsios acknowledges the CSIRO Office of the Chief Executive for funding his post-doctoral position.

\section{References}

[1] K. Bryan and J. L. Lewis. A water mass model of the global ocean. J. Geophys. Res., 84:2503-2517 (1979). doi:10.1029/JC084iC05p02503 $\mathrm{C} 461$ 
[2] J. S. Frederiksen. Precursors to blocking anomalies: the tangent linear and inverse problems. J. Atmos. Sci., 55:2419-2436 (1998). doi:10.1175/1520-0469(1998)055<2419:PTBATT>2.0.CO;2 C462

[3] J. S. Frederiksen. Self-energy closure for inhomogeneous turbulence and subgrid modeling. Entropy, 14:769-799 (2012). doi:10.3390/e14040769 C460

[4] J. S. Frederiksen and S. M. Kepert. Dynamical subgrid-scale parameterizations from direct numerical simulations. J. Atmos. Sci., 63:3006-3019 (2006). doi:10.1175/JAS3795.1 C462, C463, C472

[5] P. R. Gent and J. C. McWilliams. Isopycnal mixing in ocean circulation models. J. Phys. Oceanogr., 20:150-155 (1990). doi:10.1175/1520-0485(1990)020<0150:IMIOCM > 2.0.CO;2 C461

[6] S. M. Griffies, A. Gnanadesikan, K. W. Dixon, J. P. Dunne, R. Gerdes, M. J. Harrison, A. Rosati, J. L. Russell, B. L. Samuels, M. J. Spelman, M. Winton, and R. Zhang. Formulation of an ocean model for global climate simulations. Ocean Science, 1:45-79 (2005). doi:10.5194/os-1-45-2005 C461

[7] V. Kitsios, J. S. Frederiksen, and M. J. Zidikheri. Scaling laws for parameterisations of subgrid eddy-eddy interactions in simulations of oceanic circulations. Ocean Modelling, in print (2013). C461, C462, C465, C468

[8] J. N. Koshyk and K. Hamilton. The horizontal kinetic energy spectrum and spectral budget simulated by a high-resolution troposphere-stratosphere-mesosphere GCM. J. Atmos. Sci., 58:329-348 (2001). doi:10.1175/1520-0469(2001)058<0329:THKESA > 2.0.CO;2 C461

[9] M. H. Redi. Oceanic isopycnal mixing by coordinate rotation. J. Phys. Oceanogr., 12:1154-1158 (1982). doi:10.1175/1520-0485(1982)012<1154:OIMBCR>2.0.CO;2 C461 
[10] M. J. Zidikheri and J. S. Frederiksen. Stochastic modelling of unresolved eddy fluxes. Geophysical and Astrophysical Fluid Dynamics, 104:323-348 (2010). doi:10.1080/03091921003694701 C462, C465

[11] M. J. Zidikheri and J. S. Frederiksen. Stochastic subgrid-scale modelling for non-equilibrium geophysical flows. Phil. Trans. Royal Soc. A, 368:145-160 (2010). doi:10.1098/rsta.2009.0192 C462

\section{Author addresses}

1. V. Kitsios, Centre for Australian Weather and Climate Research (CAWCR), CSIRO Marine and Atmospheric Research, Aspendale 3195, Australia.

2. J. S. Frederiksen, CAWCR, CSIRO Marine and Atmospheric Research, Aspendale 3195, Australia.

3. M. J. Zidikheri, CAWCR, Bureau of Meteorology, Melbourne, Victoria, Australia. 